\title{
EVIDENCIA DEL COMPORTAMIENTO CIRCADIANO DE LA EPO CON DEPENDENCIA DEL SEXO E INDEPENDENCIA DE PARÁMETROS FISIOLÓGICOS EN JÓVENES ENTRENADOS EN LA ALTURA MODERADA
}

\author{
Alain Riverosi, Jesús Armando Sánchez Godor', Natalia Buitrago \\ EDGAR CRISTANCHO'.
}

\begin{abstract}
${ }^{1}$ Docente Fisiología y Líder Grupo CANNON. Facultad de Medicina; Universidad Militar Nueva Granada
${ }^{2}$ Docente Fisiología Facultad de Medicina; Universidad Militar Nueva Granada

${ }^{3}$ Estudiante Línea de Investigaciones Fisiológicas Facultad de Medicina; Universidad Militar Nueva Granada

${ }^{4}$ Docente e Investigador Fisiología Líder Grupo de Investigación en Adaptaciones al Ejercicio y a la Hipoxia
\end{abstract}

Correspondencia: alain.riveros@unimilitar.edu.co

Recibido: Abril 1 de 2014 Aceptado: Julio 30 de 2014

\begin{abstract}
Resumen
La importancia de la Epo como hormona central en los procesos de ambientación a la altura es clara dado su efecto estimulante sobre la producción eritrocitaria. Su naturaleza hormonal ha llevado a que algunas investigaciones evalúen su circadianidad con reportes no contundentes e inclusive contradictorios al respecto.

Objetivo: Analizar la circadianidad de la Epo en sujetos que realizan entrenamiento aeróbico en la altura intermedia (2600 msnm) comparando diferencias entre hombres y mujeres.

Materiales y métodos: Se reclutaron residentes a la altura intermedia de por lo menos 18 meses, de ambos sexos y que tuvieran entrenamiento aeróbico al menos durante 8 meses previos a la realización del estudio para medir su Epo cada 4 horas durante 24 horas y correlacionarla con algunas variables fisiológicas y ambientales.

Resultados: Existe comportamiento circadiano en la Epo tanto para hombres como para mujeres, con un mesor para hombres de 20:10 h y 16:33 h para mujeres. En cuanto a la acrofase, esta se presenta hacia las 17:52 h para hombres y a las 15:50 h para las mujeres. El análisis de diferencia de medias muestra una diferencia estadísticamente significativa entre los dos grupos

Conclusión: Existe circadianidad diferencial entre hombres y mujeres en cuanto a sus niveles de Epo, la cual es independiente de variables fisiológicas y ambientales.

Tema: Ritmo circadiano.

Subtema: Eritropoyetina.

Palabras claves: Ritmo circadiano, eritropoyetina, ambientación, altura intermedia.
\end{abstract}




\title{
SEX DEPENDENT AND PHYSIOLOGICALLY INDEPENDENT CIRCADIAN VARIATION IN EPO IN YOUNG AMATEUR ATHLETES
}

\begin{abstract}
Erythropoietin Epo is the key hormone in the process of erythropoiesis and, therefore, essential for acclimation to altitude. Although circadian variation in Epo levels has been reported before, the results are often contradictory. Here, we tested the hypothesis that Epo levels follow a circadian variation and that such variation depends on gender and standard physiological variables. We evaluated circadian variation in Epo levels in amateur young athletes living at intermediate elevation (Bogota, Colombia: $2600 \mathrm{~m}$ ) and analyzed the potential association with physiological variables. Materials and Methods: Six men and six women were included in the study assuring at least eight months of aerobic regime before participation. We took blood samples every $4 \mathrm{~h}$ over $24 \mathrm{~h}$ to quantify Epo levels and measured physiological variables (hear rate, blood pressure, oxygen saturation).

Results: Our results show a circadian variation in Epo levels (cosinor analysis $\mathrm{p}<0.05$ ) and a significant difference in mesor ( $\mathrm{men}=20: 10 \mathrm{~h}$, women $=16: 33 \mathrm{~h}, \mathrm{p}=0.0001$ ) and acrophase between males and females (men $=17: 52 \mathrm{~h}$, women $=15: 50 \mathrm{~h}$ ). In contrast, we did not detect any significant association with the physiological variables measured.

Conclusion: We conclude that there is a circadian variation in Epo levels and that such rhythmicity is dependent on gender and independent of physiological variables. Ongoing research aims to detect the controller of the oscillation detected in this project.

Themes: Circadian Rhythm.

Topics: Erythropoietin.

Keywords: Circadian Rhythm, Erythropoietin, Acclimation, Intermediate altitude.

\section{EVIDENCIA DO COMPORTAMENTO CIRCADIANO DA EPO COM DEPENDÊNCIA DO SEXO E INDEPENDÊNCIA DE PARÂMETROS FISIOLÓGICOS EM JOVENS TREINADOS NA ALTURA MODERADA}

\footnotetext{
Resumo

A importância da Epo como hormona central nos processos de adaptação na altura é evidente dado o efeito estimulante sobre a produção eritrocitária. Sua natureza hormonal tem levado à pesquisa da circadianidade desta, com resultados não contundentes e inclusive contraditórios.

Objetivo: Analisar de forma comparativa a circadianidade da Epo em homens e mulheres que fazem treinamento aeróbico na altura intermedia (2600 msnm).

Materiais e Métodos: Foram incluídos residentes na altura intermedia, com uma idade mínima de 18 meses, ambos sexos, e treinamento aeróbico durante pelo menos 8 meses prévios da realização da investigação. Na população incluída, a Epo foi medida com intervalo de 4 horas (durante 24 horas) com o fim de correlaciona-la com algumas variáveis fisiológicas e ambientais. Resultados: Existe comportamento circadiano na Epo tanto em homens (mesor: 20:10 h) quanto em mulheres (mesor: 16:33 h). A acrofase se apresentou às $17: 52 \mathrm{~h}$ nos homens $e$ às $15 ; 50 \mathrm{~h}$ nas mulheres. A análise comparativa de medidas descreve uma diferença estatisticamente significativa entre os dois grupos avaliados.

Conclusão: Existe circadianidade diferencial entre homens e mulheres nos níveis da Epo, sendo independente das variáveis fisiológicas e ambientais.

Tópico: Ritmo circadiano

Subtópico: Eritropoietina

Palavras-chave: Ritmo circadiano, eritropoietina, adaptação, altura intermedia.
} 


\section{Introducción}

Historia: En 1890 el anatomista francés Francois- Gilbert Viault describió por primera vez la relación entre el contenido de $\mathrm{O}_{2}$ de la sangre y la eritropoyesis al observar un aumento en el número de glóbulos rojos al ascender al cerro Morococha en Perú (aprox. 4500 msnm). Luego de permanecer 23 días en este sitio encontró que el número de eritrocitos había aumentado de 5 a 8 millones por litro de sangre ${ }^{1}$. Desde entonces varios investigadores han plateado mecanismos que explican este comportamiento como por ejemplo Friedrich Miescher en 1893 quien propuso que una disminución en la presión de oxígeno en la médula ósea proporcionaba un estímulo directo a las células eritroides, teoría que fue refutada medio siglo luego que se midió la saturación de oxígeno en muestras de médula ósea de pacientes con eritrocitosis tanto primaria como secundaria ${ }^{2}$. Solo hasta 1906 Carnot y Deflandre plantearon un mecanismo alternativo para la inducción hipóxica de la eritropoyesis. Ellos observaron un aumento en los recuentos de células rojas de la sangre después de la infusión de conejos normales con suero de animales anémicos y llegaron a la conclusión de que la eritropoyesis era regulada por un "factor " humoral en el plasma. Los intentos de reproducir este experimento en las décadas siguientes dieron resultados dudosos o negativos, lo que le hizo perder credibilidad a esta hipótesis. Sin embargo, en la mitad del siglo XX, Krumdieck y Erslev modificaron el diseño experimental de Carnot y Deflandre mediante la realización de mediciones precisas de reticulocitos, demostrando la inducción de nueva producción de glóbulos rojos dentro de 3 - 6 días después de la inyección del suero procedente de los conejos anémicos, por lo que concluyeron que el plasma de estos especímenes contenía un factor capaz de estimular la producción de glóbulos ro$\mathrm{jos}^{3}$, demostrándose de esta manera la existencia de la que en el futuro se denominaría la eritropoyetina (Epo).

La Epo es una glicoproteína ácida de aproximadamente $30 \mathrm{kDa}$ y comprende 165 aminoácidos y cuatro glicanos. La Epo circulante presenta varias isoformas que difieren en la carga eléctrica y la actividad biológica. Las cantidades de Epo se expresan en unidades internacionales (UI). Una unidad de Epo (U) se ha definido como la dosis que produce la misma respuesta estimulante de la eritropoyesis que la inyección de $5 \mu \mathrm{mol}$ de cloruro de cobalto en animales de experimentación. ${ }^{1}$ La Epo circula en el plasma con una vida media de 7-8 h, y se une con una alta afinidad a los receptores presentes, en cantidades relativamente pequeñas (aprox. 1000/célula), sobre la superficie de las células progenitoras eritroides en la médula ósea. El receptor experimenta un cambio conformacional frente a la unión de la Epo lo que permite la fosforilación cruzada a través de la unión de la quinasa JAK2 y la iniciación de la cascada de transducción de señales que induce la diferenciación y proliferación de las células de la línea eritroide ${ }^{45}$.

En los seres humanos y otros mamíferos, la eritropoyesis normalmente se realiza a una tasa basal baja, de manera que haya una sustitución de los glóbulos rojos senescentes con reticulocitos jóvenes. De otra parte, la producción de glóbulos rojos en los seres humanos se incrementa hasta en ocho veces respecto a la producción basal frente a situaciones clínicas como la hemorragia, la hemólisis y otros tipos de estrés que deterioran la oxigenación de la sangre arterial o la entrega de oxígeno a los tejidos ${ }^{6}$.

La Epo es la principal, y probablemente la única mediadora central de la inducción hipóxica de la eritropoyesis. Durante el desarrollo fetal, la Epo se produce principalmente en el hígado; sin embargo, después del nacimiento, el riñón genera aproximadamente el 80\% de su producción. Erslev propuso al túbulo proximal como el lugar ideal para la producción de $\mathrm{Epo}^{7}$. De hecho, los estudios de hibridación in situ han demostrado que la expresión de ARNm de la Epo en el riñón se localiza en un subconjunto de los fibroblastos peritubulares en la corteza en cercanía a la médula ${ }^{89}$. En el hígado la Epo se produce tanto en los hepatocitos como en las células intersticiales. De otra parte, la hipoxia induce un aumento de la producción de la hormona Epo en el riñón, que luego circula en el plasma y se une a los receptores abundantemente expresados en las células progenitoras eritroides, promoviendo así la viabilidad, proliferación y diferenciación de los precursores de los glóbulos rojos. Esto permite optimizar el aporte de oxígeno $\left(\mathrm{DO}_{2}\right)$ al riñón, completando así el arco de realimentación que regula la expresión de la Epo. La inducción hipóxica de la Epo depende en gran parte del factor de transcripción HIF el cual se activa en prácticamente todas las células por la exposición a la hipoxia, así como por los quelantes del cobalto o el hierro?.

\section{Variación circadiana de la función renal y de la síntesis de Epo}

Una buena parte de los procesos fisiológicos funcionan con una periodicidad de aproximadamente $24 \mathrm{~h}$. La longitud aproximada de este período dio lugar al nombre circadiano, que se compone de dos palabras latinas: circa (aproximadamente) y dies (días). Funcionalmente, se cree que los ritmos circadianos proporcionan una ventaja importante al permitir al organismo anticipar los cambios ambientales rutinarios. La base molecular de los ritmos circadianos en los mamíferos fue descrita a finales del siglo $\mathrm{XX}$. Desde entonces se demostró que el reloj circadiano de mamíferos es un sistema jerárquicamente organizado 
de osciladores celulares individuales orquestados por un marcapasos central que reside en el núcleo supraquiasmático (SCN) del hipotálamo ${ }^{10}$. El marcapaso del SCN se sincroniza con el mundo exterior, principalmente por el ciclo de luz/oscuridad. Las oscilaciones circadianas pueden modificar la trascripción génica, lo que lleva a oscilaciones funcionales a nivel tisular. Las estimaciones actuales indican que hasta un $10 \%$ de todos los genes están bajo el control de los factores transcripcionales circadianos ${ }^{11}$.

Los ritmos circadianos en la función renal se han estudiado desde mediados del siglo XIX. En 1861, Edward Smith, uno de los pioneros en la fisiología circadiana, publicó la primera evidencia documentada de la existencia de oscilaciones circadianas en la excreción renal de la urea y el agua ${ }^{12}$. Este patrón de excreción también se ha encontrado para el sodio, el potasio, el cloro y otros electrolitos $^{13}$. Dado que la mayoría de los ritmos de excreción se mantienen en pacientes con trasplante renal ${ }^{14}$, se concluye que tanto factores humorales como renales intrínsecos -aún desconocidos- están involucrados en su generación. La evidencia de la existencia de un mecanismo renal intrínseco ha sido difícil de estudiar debido a la dificultad de disociar este mecanismo de los efectos de los factores neuroendocrinos circadianos. De tal manera, se ha propuesto que el riñón posea un reloj circadiano intrínseco potencialmente implicado en el control transcripcional / traduccional de miles de genes del transcriptoma renal ${ }^{15}$.

Los estudios sobre el ritmo circadiano de la Epo son escasos a la fecha $y$, en principio, no concluyentes. Algunas investigaciones anteriores ${ }^{16,17,18}$ han detectado ritmos circadianos importantes en la Epo, mientras que otros, sólo cambios menores durante el día ${ }^{19}$, o incluso, ausencia de fluctuaciones ${ }^{20}$. $\mathrm{El}$ estudio de Klausen y cols ${ }^{18}$ se realizó con nueve sujetos, no sólo a nivel del mar, sino también a una altitud de 4350 msnm durante 4 días. Al nivel del mar, así como en la altura, la Epo mostró un marcado ritmo circadiano con un nadir entre las 08:00-16:00 h. Los resultados también sugieren que la producción de Epo en la altura es afectada por los cambios en el pH y la afinidad de la hemoglobina por el oxígeno. Una explicación preliminar de los resultados tan disímiles podría ser las diferentes configuraciones de las investigaciones. Klau$\operatorname{sen}^{18}$, por ejemplo, permitió que los sujetos se movilizaran durante el día, mientras que en la investigación de Gunga $y$ cols. los sujetos fueron completamente restringidos a una posición supina durante las $24 \mathrm{~h}^{21}$, lo que podría acarrear diferencias en el patrón de secreción a consecuencia de los cambios en la distribución de la volemia a lo largo del eje corporal. Esto último se ha demostrado que modifica de manera importante la presión venosa central.
La determinación de la actividad de Epo en hombres y mujeres con distintos niveles de entrenamiento y altura de procedencia no ha demostrado la esperada correlación positiva con variables sanguíneas como la masa de hemoglobina ${ }^{22}$. Dos hipótesis pueden explicar la falta de correlación entre las variables: por un lado, la variación circadiana en la secreción de Epo que podría afectar los análisis de muestras tomadas en distintas horas del día y por otra parte, la mínima y no significativa diferencia en la actividad de Epo registrada entre nativos de tierras bajas y de la altura moderada sería suficiente para generar las notables diferencias en hemoglobina total ( $\mathrm{HbT})$.

Este estudio establece la magnitud en que la $\mathrm{SaO}_{2}$ y la Epo se correlacionan en hombres y mujeres que realizan entrenamiento aeróbico en la altura intermedia en un día de actividad cotidiana sin entrenamiento, lo cual hasta ahora no se ha investigado y permite avanzar en el entendimiento de la respuesta fisiológica a condiciones ambientales y de estrés comparando adicionalmente la respuesta diferencial entre los sexos. Para este propósito se realizó una evaluación de diferentes parámetros fisiológicos y mediciones secuenciales de la Epo durante el día, las cuales se trataron mediante el análisis de cosinor. Dicho método fue desarrollado a principios de 1960 y basado en la premisa que los ritmos circadianos responden a un comportamiento periódico con pequeñas variaciones aleatorias que pueden ser descritas ajustando las observaciones a funciones cosinusoidales (o sinusoidales) por medio de mínimos cuadrados y en los que cada observación es una función del estimador estadístico de la línea media del ritmo ${ }^{23}$. La prueba se basa en la proyección del vector obtenido a partir de la amplitud de la función y el ángulo definido por la acrofase, de modo que si dicho vector se superpone con la región de aceptación $(p<0,05)$ se rechaza la hipótesis nula de no circadianidad.

\section{Materiales y métodos}

\section{Sujetos}

Este estudio incluyó 6 hombres y 6 mujeres (Tabla 1). Se consideraron como criterios de exclusión la presencia de enfermedades renales, cardiacas, hematológicas o pulmonares, y ser fumador o sedentario. Se consideraron como criterios de inclusión ser residente a la altura intermedia (2600 msnm) por lo menos durante 18 meses y haber realizado entrenamiento aeróbico mínimo 10 horas por semana durante los últimos 8 meses. Todos los individuos firmaron el consentimiento informado siendo el protocolo aprobado por el Comité de Investigaciones de la Universidad Militar Nueva Granada. 
Tabla 1. Comparación de datos antropométricos y hemograma entre hombres y mujeres. BMI: Índice de masa corporal, RBC: conteo de eritrocitos, HB: Hemoglobina, MCV: Volumen corpuscular medio, MHC: Hemoglobina corpuscular media, HT: Hematocrito, MCHC: Concentración de la hemoglobina corpuscular media, RDW: Ancho de distribución eritrocitaria, X: Promedio, SD: Desviación estándar.

\begin{tabular}{|c|c|c|c|c|c|c|}
\hline & \multicolumn{2}{|c|}{ Hombres } & \multicolumn{2}{|c|}{ Mujeres } & \multirow{2}{*}{ Unidades } & \multirow{2}{*}{$\mathbf{p}$} \\
\hline & $\mathbf{X}$ & SD & $\mathbf{X}$ & SD & & \\
\hline EDAD & 20,2 & 1,72 & 19,8 & 0,75 & Años & 0,68 \\
\hline MASA & 70,6 & 7,27 & 53,2 & 3,44 & $\mathrm{~kg}$ & $<0,01$ \\
\hline TALLA & 1,8 & 0,04 & 1,6 & 0,06 & $\mathrm{~m}$ & $<0,01$ \\
\hline BMI & 22,8 & 1,72 & 20,7 & 0,82 & $\mathrm{~kg} / \mathrm{m}^{2}$ & 0,03 \\
\hline $\mathrm{RBC}$ & 5,2 & 0,31 & 5 & 0,26 & $\mathrm{X} 10^{6} \mathrm{cel} / \mu \mathrm{L}$ & 0,14 \\
\hline $\mathrm{HB}$ & 16,4 & 0,92 & $15,4-$ & 0,83 & $\mathrm{~g} / \mathrm{dL}$ & 0,02 \\
\hline HT & 48,6 & 2,48 & 45,3 & 1,45 & $\%$ & 0,07 \\
\hline $\mathrm{MCV}$ & 93,2 & 1,48 & 91,5 & 3,25 & $\mathrm{fL}$ & 0,28 \\
\hline $\mathrm{MCH}$ & 31,5 & 0,79 & 31 & 0,88 & $\mathrm{pg}$ & 0,41 \\
\hline $\mathrm{MCHC}$ & 33,7 & 0,39 & 33,9 & 0,77 & $\mathrm{~g} / \mathrm{dL}$ & 0,57 \\
\hline RDW & 12,9 & 0,47 & 12,7 & 0,39 & $\%$ & 0,41 \\
\hline
\end{tabular}

\section{Mediciones hematológicas}

La sangre fue colectada de la vena del antebrazo con un tubo de plástico al vacío Vacuette ${ }^{\circledR}$ que contenía $1,2-2 \mathrm{mg}$ de K3-EDTA. Cada 4 horas se realizó la extracción de 3 a $4 \mathrm{~mL}$ de sangre, siendo la muestra inicial tomada al medio día del día 1 de investigación. Esa primera muestra también sirvió para la medición de las variables hematológicas del cuadro hemático estándar. Durante las 24 horas que se evaluó, y por lo menos las 8 horas previas, los individuos no realizaron ningún tipo de entrenamiento físico.

\section{Método para la medición de la Epo}

Una vez obtenidas las muestras de sangre, fueron separados los plasmas de las fases celulares por sedimentación y almacenados a $4^{\circ} \mathrm{C}$. Una vez colectadas todas las muestras, fueron liofilizadas y resuspendidas en agua destilada para realizar la medición de eritropoyetina con el EPO-ELISA kit (Roche ${ }^{\circledR}$ Diagnostics $\mathrm{GmbH}$ ). Las muestras, los controles y los estándares fueron dispuestos en sendos pozos de una placa de dilución a la cual fue agregado el buffer del kit. Una vez estabilizadas las soluciones, estas fueron servidas en microplatos con pozos cubiertos por anti-EPO policlonal de conejo. Los microplatos fueron dispuestos en una incubadora de $\mathrm{CO}_{2} \mathrm{VWR}^{\circledR}$ a $37^{\circ} \mathrm{C}$ sobre un mezclador ShakerDuo Max 1030 Heidolph $^{\circledR}$ a 300 cpm siguiendo el protocolo del kit. Una vez terminada la fase de reacción se realizó la medición de absorbancia a $450 \mathrm{~nm}$ con un lector de placas (Pioway ${ }^{\circledR}$ MB1008056).Los valores de absorbancia fueron tabulados y analizados en Microsoft Exce ${ }^{\circledR}$ (2013, Redmond, WA) en el cual se realizó una interpolación usando una regresión potencial para obtener la curva de calibración y determinar las concentraciones.

\section{Mediciones ambientales}

Dada las implicaciones de los fenómenos ambientales sobre los procesos fisiológicos evaluados durante este estudio se realizó una monitorización periódica de las siguientes variables: punto rocío (DP), presión barométrica (BP), humedad $(\mathrm{H})$, índice de calor $(\mathrm{HI})$ y temperatura $(\mathrm{T})$. Las mediciones se realizaron con el Kestrel ${ }^{\circledR} 4500$ Pocket Weather Tracker en Bogotá a 2625 msnm y en las coordenadas $4^{\circ} 35^{\prime} 56^{\prime \prime}$ N, 74 4' $51^{\prime \prime} \mathrm{W}$.

\section{Mediciones fisiológicas}

Cada 4 horas se realizó una medición digital de signos vitales (PROPAQ CS WelchAllyn ${ }^{\circledR}$ modelo 242) que incluyó presión arterial sistólica (PAS), presión arterial diastólica (PAD), frecuencia cardiaca $(\mathrm{FC})$ y saturación arterial de oxígeno $\left(\mathrm{SaO}_{2}\right)$. Adicionalmente, y durante el periodo de sueño, se realizó monitorización permanente de la $\mathrm{SaO}_{2}$ utilizando el pulsoxímetro MLT321 (ADInstruments ${ }^{\circledR}$, Dunedin New Zealand) cuyos datos fueron tabulados en el programa LabChart ${ }^{\circledR}$.

\section{Representación gráfica y métodos estadísticos:}

Para el análisis inicial de los datos y algunas de las representaciones gráficas se utilizó el programa OriginPro ${ }^{\circledR}$ (OriginLab, Northampton, MA), mostrando el promedio $(\overline{\mathrm{X}})$ y su desviación estándar (SD), a menos que sea expresado lo contrario. La determinación de normalidad se realizó utilizando la prueba de Shapiro-Wilk los resultados con $\mathrm{p}<0,05$ fueron analizados con la prueba de Friedman mientras que si la p>0,05 se utilizó el ANOVA de medidas repetidas. 
Con el propósito de comprobar si existe un comportamiento circadiano se realizó un análisis de cosinor sobre los valores obtenidos de la Epo usando el paquete "psych" 24 para $\mathrm{R}^{25}$ (versión 3.0.2). Para verificar si la distribución de la variable Epo corresponde a una distribución circular de Von Mises, se realizó un test de Watson ${ }^{26}$ sobre los valores de la Epo. Esta prueba se realizó usando el paquete "circular" ${ }^{27}$ para R. Los valores de MESOR, amplitud, acrofase y la varianza circular fueron obtenidos para cada sexo. Usando el programa PAST ${ }^{28}$ y los datos de MESOR y varianza circular obtenidos con "psych", se realizó una prueba $T$ entre las fases de ambos grupos y una prueba $F$ para evaluar diferencias entre sus varianzas circulares siguiendo la metodología de Atkinson y cols ${ }^{29}$.

El comportamiento diario de la Epo fue graficado para hombres y mujeres por medio de diagramas de cajas y bigotes para cada sexo además de un diagrama conjunto utilizando R. Adicionalmente generamos gráficas de distribución circular de los valores de eritropoyetina medidos en hombres y mujeres usando los paquetes "plotrix", "glm", "circular" y "ggplot" para R.

\section{Resultados}

Descripción general de la población. La tabla 1 resume las principales variables antropométricas en las cuales se aprecia $\overline{\mathrm{X}}$ y SD en cada grupo y la prueba T con una significancia estadística de p<0,05 que analizó la diferencia entre grupos. Se muestran también las principales variables del cuadro hemático. Aunque se trató de grupos etarios similares, hubo diferencias significativas para el peso, la talla y el índice de masa corporal, lo cual se corresponde con el dimorfismo sexual propio de la especie humana ${ }^{30}$. En cuanto al cuadro hemático, es llamativo que solo pare- ce existir diferencia significativa entre los dos grupos para la concentración de hemoglobina, lo cual contrasta con estudios poblacionales que analizan la diferencia entre sexos 31,32 . Aunque no son presentados los resultados de la línea blanca y plaquetas, estos fueron tenidos en cuenta para descartar procesos inflamatorios o alteraciones celulares no inferidas por la historia clínica.

Condiciones ambientales. En la figura 1 se puede apreciar el comportamiento de las principales variaciones de los parámetros ambientales de los días de prueba. Dichos valores fueron analizados estadísticamente utilizando ANOVA de medidas repetidas después de verificar que correspondieran a una distribución normal, encontrándose que no hay diferencias significativas en el comportamiento de los mismos a lo largo del día (ver tabla 2). Se puede apreciar entonces que las variables ambientales mostraron un comportamiento relativamente estable a lo largo del día, por lo que se descarta su participación como generadores de la oscilación en las mediciones de la [Epo].

Evaluación fisiológica. La figura 2 muestra los resultados obtenidos tras la evaluación de las diferentes variables fisiológicas consideradas en este estudio. Aunque existe una leve oscilación de cada variable de acuerdo a la hora del día, este patrón parece ajustarse más a la rutina de actividad física diaria, ya que los análisis estadísticos no mostraron diferencias estadísticamente significativas al comparar mediciones de las diferentes horas del día. La tabla 3 resume dichos hallazgos en los que, exceptuando la $\mathrm{SaO}_{2} \mathrm{~m}$, no se encontraron diferencias estadísticamente significativas en dichas variables.

Evaluación de la Eritropoyetina. El comportamiento de la eritropoyetina presenta cambios importantes con respecto a la hora del día tanto para hombres como para

Tabla 2. Valores promedio para cada día y promedio general de cada variable ambiental. Valores $F$ y $p$ provenientes de ANOVA de medidas repetidas para la evaluación de diferencias entre las variables ambientales para las mediciones diarias en cada una de ellas. DP: Punto de rocío $\left({ }^{\circ} \mathrm{C}\right), \mathrm{BP}$ : Presión barométrica ( $\left.\mathrm{mmHg}\right), \mathrm{HI}$ : Índice de calor $\left({ }^{\circ} \mathrm{C}\right), \mathrm{H}$ : Humedad (\%), T: Temperatura $\left({ }^{\circ} \mathrm{C}\right)$..

\begin{tabular}{|c|c|c|c|c|c|c|c|}
\hline & \multirow[t]{2}{*}{ Día 1} & \multirow[t]{2}{*}{ Día 2} & \multirow[t]{2}{*}{ Día 3} & \multirow[t]{2}{*}{ Día 4} & \multirow[t]{2}{*}{$\begin{array}{c}\text { Promedio } \\
\text { general }\end{array}$} & \multicolumn{2}{|c|}{$\begin{array}{c}\text { Diferencias medidas } \\
\text { diarias }\end{array}$} \\
\hline & & & & & & $\mathbf{F}$ & p valor \\
\hline DP & 9,78 & 9,25 & 9,97 & 10,77 & 9,94 & 1,859 & 0,231 \\
\hline $\mathrm{BP}$ & 553 & 554 & 552 & 552 & 553 & 3,678 & 0,113 \\
\hline $\mathrm{HI}$ & 20,9 & 18,3 & 19,3 & 19,1 & 19,4 & 5,67 & 0,063 \\
\hline $\mathrm{H}$ & 50,8 & 52,7 & 52 & 56 & 52,9 & 8,437 & 0,034 \\
\hline $\mathrm{T}$ & 21,1 & 19 & 20,2 & 19,7 & 20 & 3,976 & 0,103 \\
\hline
\end{tabular}



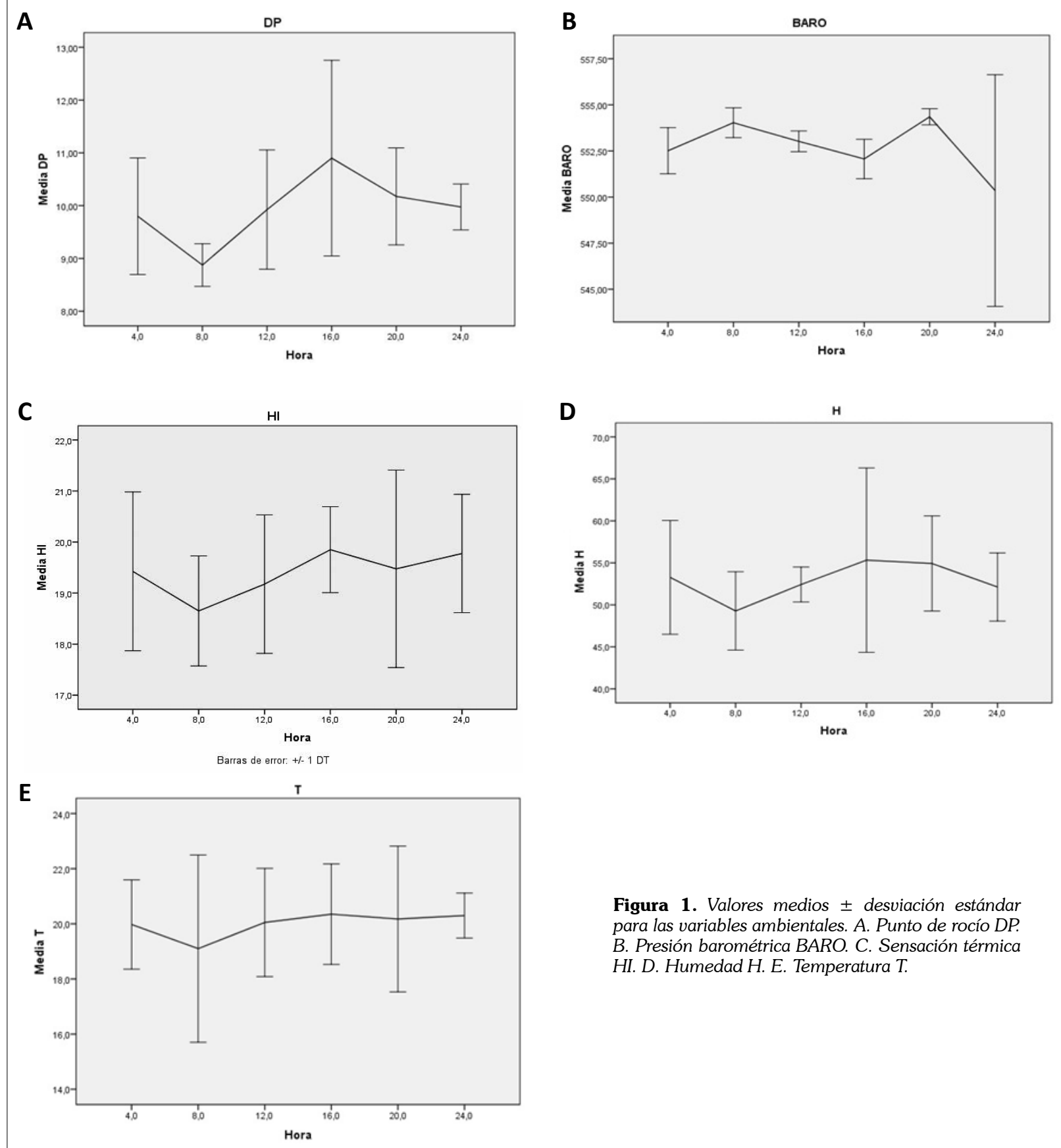

Figura 1. Valores medios \pm desviación estándar para las variables ambientales. A. Punto de rocío DP. B. Presión barométrica BARO. C. Sensación térmica HI. D. Humedad H. E. Temperatura T.

mujeres. La figura 3 muestra dicho comportamiento en el que se puede apreciar un pico hacia las 16:00 h para los dos grupos. El análisis de la diferencia en cada grupo a lo largo del día fue realizado con la prueba de Friedman la cual arrojó un valor p menor a 0,01 tanto en el grupo de hombres como en el grupo de mujeres. Para el análisis post hoc se utilizó la prueba de Wilcoxon la cual arrojó diferencias estadísticamente significativas con $\mathrm{p}=0,03$ para la mayoría de apareamientos entre las diferentes horas del día tanto en hombres como mujeres.

Para la evaluación de circadianidad se realizó la prueba de cosinor la cual demostró que la Epo tiene un comportamiento circadiano $(p<0,05)$. El test de Watson 
Tabla 3. Resultados de las pruebas estadísticas para las variables fisiológicas. Exceptuando la PADh que no tuvo un comportamiento normal y que fue analizada por la prueba de Friedman, todas las demás variables fueron analizadas con el ANOVA de medidas repetidas. Cuando la prueba de Mauchly demuestra esfericidad se reportan los valores $F$ y p en las columnas respectivas. Si se viola el supuesto de esfericidad se muestra la corrección de Greenhouse-Geisser con su $\mathrm{F}$ y p respectivos. $\mathrm{SaO}_{2} \mathrm{~h}$ : saturación arterial de oxígeno en hombres, SaO $m$ : saturación arterial de oxígeno en mujeres, PASh: presión arterial sistólica en hombres, PADh: presión arterial diastólica en hombres, PASm: presión arterial sistólica en mujeres, PADm: presión arterial diastólica en mujeres, FCh: frecuencia cardiaca en hombres, FCm: frecuencia cardiaca en mujeres.

\begin{tabular}{|l|c|c|c|c|c|c|c|}
\hline $\begin{array}{c}\text { Variable } \\
\text { fisiológica }\end{array}$ & $\begin{array}{c}\text { Prueba de } \\
\text { Shapiro- } \\
\text { Wilk }\end{array}$ & $\begin{array}{c}\text { Prueba de } \\
\text { Mauchly }\end{array}$ & $\begin{array}{c}\text { F } \\
\text { Asumiendo } \\
\text { esfericidad }\end{array}$ & $\begin{array}{c}\text { p } \\
\text { Asumiendo } \\
\text { esfericidad }\end{array}$ & $\begin{array}{c}\text { F } \\
\text { Corrección de } \\
\text { Greenhouse- } \\
\text { Geisser }\end{array}$ & $\begin{array}{c}\text { p } \\
\text { Corrección de } \\
\text { Greenhouse- } \\
\text { Geisser }\end{array}$ & $\begin{array}{c}\text { p } \\
\text { Friedman }\end{array}$ \\
\hline $\mathrm{SaO}_{2} \mathrm{~h}$ & 0,19 & 0,05 & & & 0,86 & 0,44 \\
\hline $\mathrm{SaO}_{2} \mathrm{~m}$ & 0,08 & 0,78 & 2,70 & 0,04 & & \\
\hline $\mathrm{PASh}$ & 0,80 & 0,40 & 1,20 & 0,34 & & \\
\hline $\mathrm{PADh}$ & $<0,01$ & & & & & \\
\hline $\mathrm{PASm}$ & 0,87 & 0,29 & 2,22 & 0,08 & & \\
\hline $\mathrm{PADm}$ & 0,90 & 0,02 & & & & \\
\hline $\mathrm{FCh}$ & 0,83 & 0,72 & 2,50 & 0,06 & & \\
\hline $\mathrm{FCm}$ & 0,30 & 0,45 & 1,56 & 0,21 & & \\
\hline
\end{tabular}

A

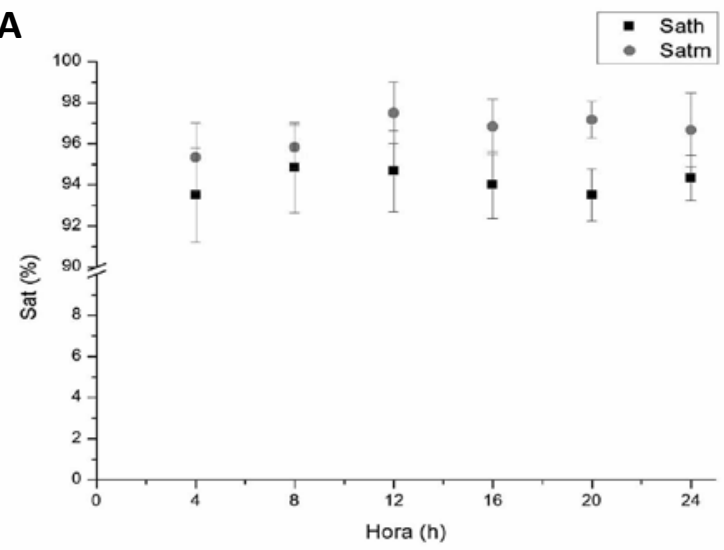

C

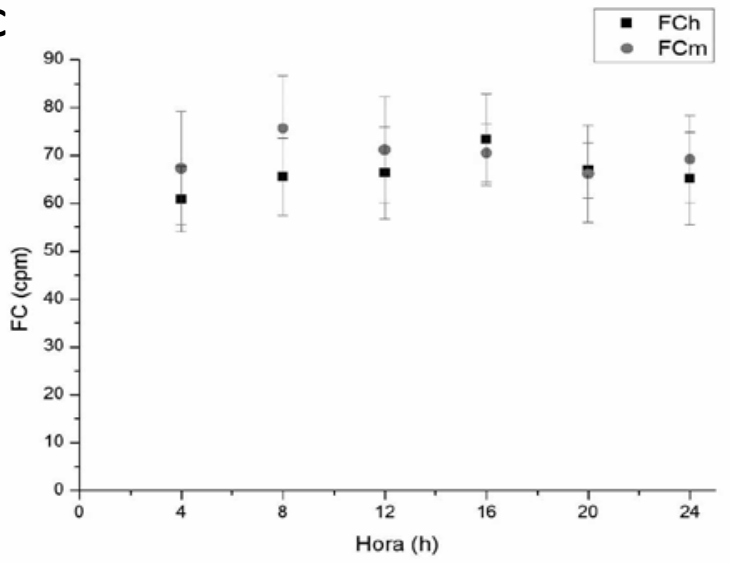

B

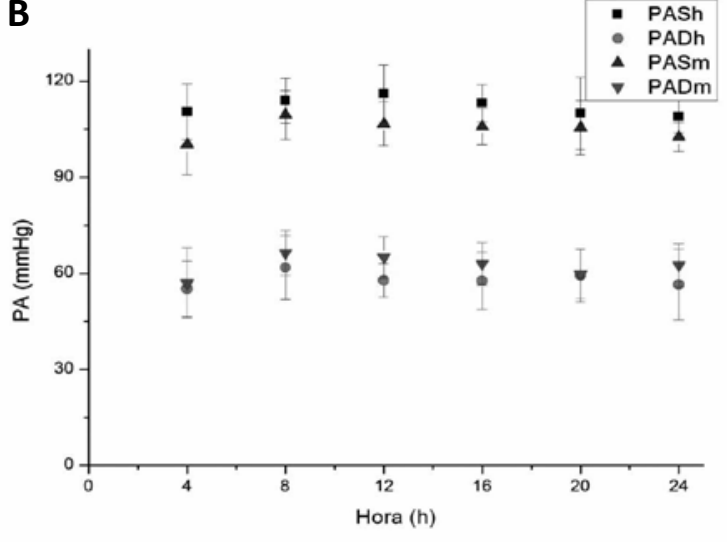

Figura 2. Comportamiento de las variables fisiológicas a lo largo del día. A. Porcentaje de saturación arterial de oxígeno $\left(\mathrm{SaO}_{2}\right)$. B. Presión arterial en $\mathrm{mmHg}(\mathrm{PA})$. C. Frecuencia cardiaca en ciclos por minuto. $\mathrm{SaO}_{2} \mathrm{~h}$ : saturación arterial de oxígeno en hombres, $\mathrm{SaO}_{2} \mathrm{~m}$ : saturación arterial de oxígeno en mujeres, PASh: presión arterial sistólica en hombres, PADh: presión arterial diastólica en hombres, PASm: presión arterial sistólica en mujeres, PADm: presión arterial diastólica en mujeres, FCh: frecuencia cardiaca en hombres, FCm: frecuencia cardiaca en mujeres. 

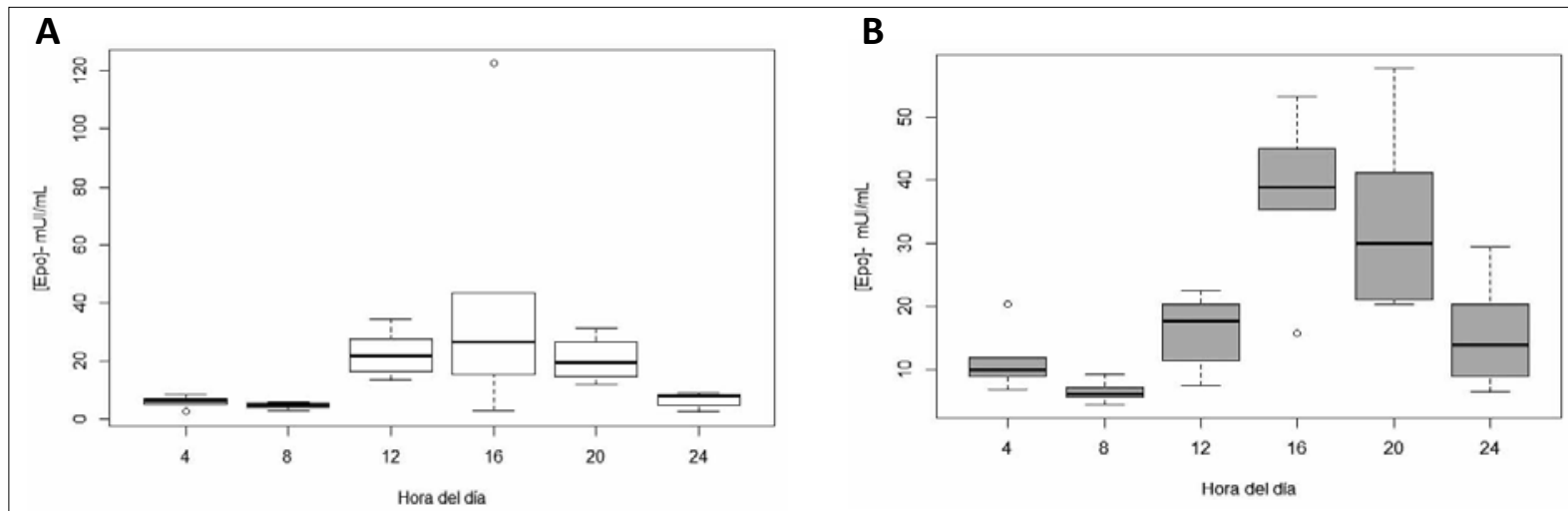

C

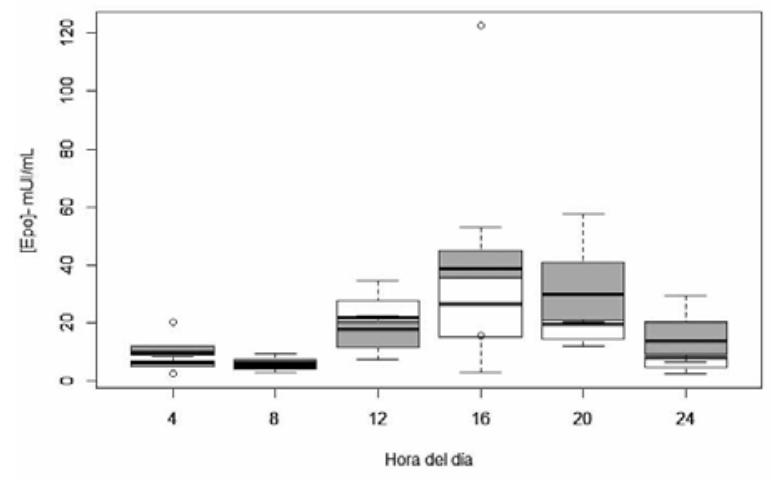

Figura 3. Gráficos de cajas y bigotes describiendo el comportamiento general de la variable Epo en cada una de las horas del día en que se realizaron los registros. A. Variación en mujeres. B. Variación en hombres. C. Gráfico comparativo entre hombres (cajas grises) y mujeres (cajas blancas).

mostró que los datos no corresponden a una distribución de Von Mises ( $p<0,117)$, por lo que no se utilizaron pruebas circulares. La prueba $F$ mostró que no existen diferencias significativas en términos del MESOR para hombres y mujeres ( $F=2,2988 ; p=0,38)$, mientras que la prueba $T$ para dos muestras con igual varianza mostró que existe una diferencia significativa entre los MESORES de ambos grupos $(T=6,1628 ; p<0,05)$. Se encontraron diferencias significativas en cuanto a la amplitud y la acrofase para ambos grupos. La figura 4 muestra el resumen de dicho análisis y la representación gráfica de las diferencias en amplitud y acrofase de ambos grupos.

\section{Discusión}

Este estudio evidencia la existencia de un ritmo circadiano en los niveles circulantes de la Epo en adultos jóvenes deportistas sanos que realizan actividades cotidianas durante un día sin entrenamiento, tanto en hombres como en mujeres. Esto controvierte los hallazgos publicados por Roberts y cols. en 1996 quien estudió a 26 hombres sanos (edad $27 \pm 4$ años) que no estaban vinculados a un programa de entrenamiento físico ${ }^{20}$. De igual manera, en nuestro estudio se encontró un patrón circadiano en su-

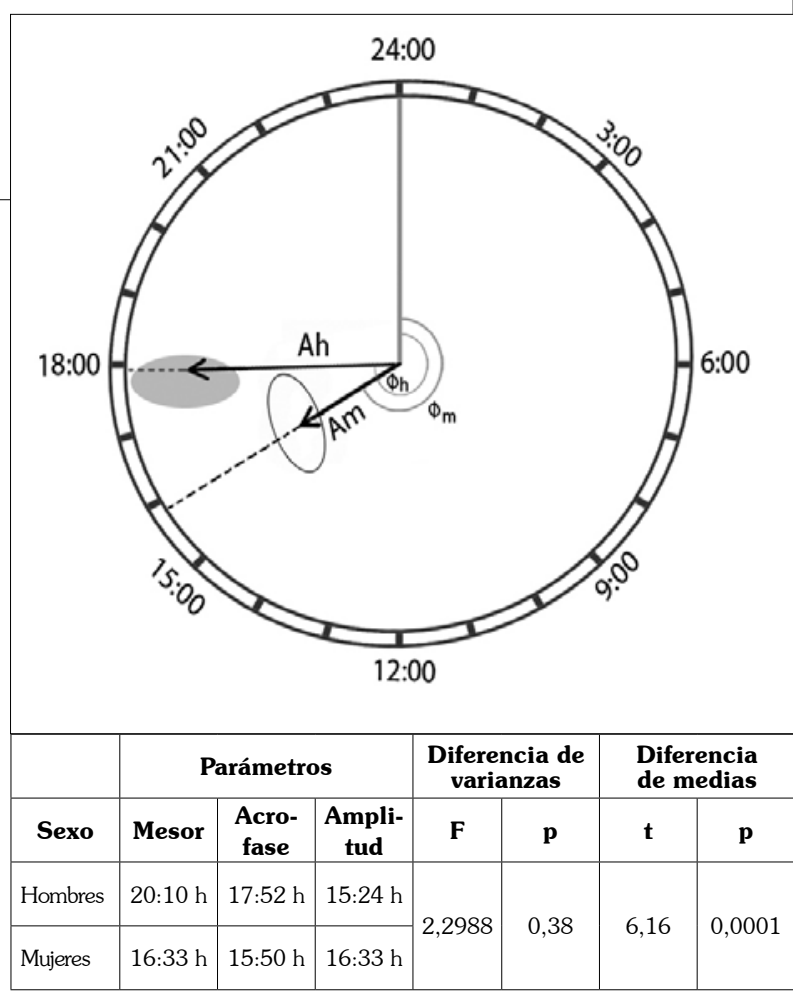

Figura 4. A) Diagrama circular mostrando los valores de acrofase media de hombres $(\varphi h)$ y mujeres $(\varphi m)$; La amplitud media de hombres (Ah) y mujeres (Am) y los intervalos de confianza de ambos valores para hombres (elipse gris) y mujeres (elipse blanco) para Epo en un periodo de 24 horas. Parámetros de las funciones cosinor para hombres y mujeres; estadísticos de prueba $\mathrm{F}$ y $\mathrm{t}, \mathrm{y}$ valores $\mathrm{p}$ describiendo la existencia de diferencias significativas entre hombres y mujeres. 
jetos sanos a diferencia de lo resultados de Miller y cols. ${ }^{19}$ quienes midieron los niveles de Epo cada 4 h durante 24 h en 17 sujetos hematológicamente normales, así como en 30 sujetos con enfermedad pulmonar crónica. Estos investigadores encontraron que sólo los pacientes con enfermedad pulmonar crónica mostraban una variación diurna con un cenit en la medianoche, sin variación significativa en los sujetos normales ${ }^{19}$. Las diferencias pueden radicar en el tipo de análisis estadístico utilizado ya que Roberts y cols. utilizaron un análisis multivariado para evaluar las diferencias de los niveles de la Epo individualmente y entre grupos, mientras que Miller y cols. publicaron sus resultados sin una clara demostración estadística de la ausencia o presencia de cambios diurnos en las concentraciones de $\mathrm{Epo}^{19}$. En el presente estudio además de encontrar diferencias en los promedios de las concentraciones cada 4 horas por medio de pruebas no paramétricas, en ambos sexos, se evidenció la presencia de un ritmo circadiano en ambos grupos a través del análisis de cosinor. Este método es capaz de detectar la existencia de un ritmo circadiano significativo y los parámetros de dicho ritmo: mesor (nivel promedio de ritmo), amplitud (longitud de MESOR a NADIR) y acrofase (pico de ritmo) ${ }^{33}$.

El patrón circadiano en la Epo no se modifica con la edad. Así, en el estudio realizado por Pasqualetti y cols. a finales del siglo $\mathrm{XX}^{34}$ no encontraron diferencias significativas entre dos poblaciones, una con una edad promedio de 43,3 $\pm 8,3$ años y otra de $65,3 \pm 6,4$ años. Los hallazgos de Pasqualetti y cols. se complementan con los encontrados en nuestro estudio ya que estudiamos una población de adultos jóvenes con promedio de edad de 20,2 $\pm 1,7$ años. $\mathrm{Al}$ igual que en el presente estudio, Pasqualetti y cols utilizaron el análisis de cosinor y también se encontró una acrofase alrededor de las 18:00 h en hombres y 16:00 h en mujeres. Aunque se han descrito diferencias de acuerdo al método de medición de la Epo, ${ }^{35}$ los hallazgos del presente estudio son consistentes con otros estudios en los que utilizó el radioinmunoensayo ${ }^{17,36,34,37}$.

Aunque el principal estímulo para la producción de la Epo es la hipoxia, esta no explica el pico encontrado entre las 16:00 y las 20:00 horas. De hecho, no se encontró relación alguna entre la $\mathrm{SatO}_{2}$ y la Epo como se ha descrito previamente ${ }^{36}$. Los mecanismos que explican este patrón circadiano aún son desconocidos, sin embargo se ha encontrado que este patrón no es exclusivo para esta molécula de origen renal, sino que también se encuentra en otros procesos renales relacionados con el manejo tubular de solutos. ${ }^{15,38}$ Adicionalmente, se ha planteado que se debe considerar que las variaciones diurnas en el flujo sanguíneo renal podrían explicar las fluctuaciones existentes tanto en la tasa de secreción como en el metabolismo de la $\mathrm{Epo}^{17}$.

Cabe destacar con respecto a otras publicaciones en el área que aquí fueron tenidas en cuenta y analizadas las variables ambientales. Las condiciones atmosféricas ejercen efectos importantes sobre parámetros fisiológicos que podrían estar implicados en la adaptación a la altura o que podrían convertirse en factores de confusión para un análisis de circadianidad ${ }^{39,40}$, específicamente relacionados con la presión alveolar de oxígeno y su impacto sobre la presión arterial de oxígeno. Además de la altitud es necesario tener presentes factores como la latitud o la disposición geográfica ya que pueden desencadenar cambios en variables como la presión barométrica o la temperatura. Bogotá, una ciudad ubicada en los trópicos, no tiene los cambios estacionales propios de las regiones boreales y australes; sin embargo, su continentalidad y la influencia del frente intertropical condicionan un clima voluble. En el presente estudio el análisis de algunas variables ambientales no demostró cambios significativos durante el día, lo que refuerza la idea que los cambios observados en la Epo deben ser explicados por un mecanismo intrínseco (muy probablemente de origen renal) que amerita nuevos estudios para su esclarecimiento. Esto último, probablemente a través de estudios en modelo animal donde se controle la regulación neuroendocrina de la secreción de la Epo o a través de las herramientas genéticas que permitan la fenotipificación de los ritmos circadianos de origen renal ${ }^{15,41}$.

\section{Conclusión}

Se ha mostrado evidencia de un comportamiento diferencial de la Epo a lo largo del día tanto para mujeres como para hombres, siendo en estos últimos más marcado el efecto. Se explica este comportamiento por una circadianidad evidente por análisis específico y que pone de manifiesto una acrofase del fenómeno alrededor de las 18:00 horas. El oscilador responsable de dicho comportamiento es motivo de futuras investigaciones que buscarán determinar el papel del riñón como controlador local de un fenómeno global.

Agradecimientos: El grupo de investigación reconoce además la colaboración de la doctora Diana Pachón por el procesamiento y medición de la eritropoyetina. 
Finalmente, agradecemos a las auxiliares de laboratorio Esther Jiménez y Pilar Pérez por su buena disposición para la realización de las pruebas fisiológicas e inmunológicas que requirió el trabajo.

Conflicto de intereses: Ninguno reportado por los autores.

Financiación: Este estudio fue financiado por la Universidad Militar Nueva Granada dentro de su programa de financiación a investigaciones internas bajo el proyecto MED1075.

\section{Referencias}

1. Jelkmann W. Erythropoietin after a century of research: younger than ever. Eur J Haematol 2007;78(3):183-205.

2. Stohlman F, Rath CE, Rose JC. Evidence for a humoral regulation of erythropoiesis; studies on a patient with polycythemia secondary to regional hypoxia. Blood 1954;9(7):721-33.

3. Erslev A. Evidence for a humoral regulation of erythropoiesis; studies on a patient with polycythemia secondary to regional hypoxia. Blood 1953;8(4):349-57.

4. Livnah O. Crystallographic Evidence for Preformed Dimers of Erythropoietin Receptor Before Ligand Activation. Science (80) 1999;283(5404):987-90.

5. Remy I. Erythropoietin Receptor Activation by a Ligand-Induced Conformation Change. Science (80- ) 1999; 283(5404):990-3.

6. Wenger RH, Kurtz A. Erythropoietin. Compr Physiol 2011; 1(4):1759-94.

7. Erslev A, Caro J, A B. Why the kidney? Nephron 1985;41(3): 213-6.

8. Koury ST, Koury MJ, Bondurant MC, Caro J, Graber E. Quantitation Hybridization: of Erythropoietin-Producing Correlation With Hematocrit, Serum Erythropoietin Cells in Kidneys of Mice Renal Erythropoietin Concentration. Blood 1989;74(2):645-51.

9. Maxwell PH, Pugh CW, Ratcliffe PJ. Inducible operation of the erythropoietin 3' enhancer in multiple cell lines: evidence for a widespread oxygen-sensing mechanism. Proc Natl Acad Sci U S A 1993;90(6):2423-7.

10. Schibler U, Ripperger J, Brown SA. Peripheral Circadian Oscillators in Mammals: Time and Food. J Biol Rhythms 2003;18(3):250-60

11. Buhr ED, Takahashi JS. Molecular components of the Mammalian circadian clock. Handb Exp Pharmacol 2013;(217):327.

12. Smith E. Health and disease: as influenced by the daily, seasonal, and other cyclical changes in the human system. London: Walton and Maberly; 1861.

13. Hurwitz S, Cohen RJ, Williams GH. Diurnal variation of aldosterone and plasma renin activity: timing relation to melatonin and cortisol and consistency after prolonged bed rest. J Appl Physiol 2004;96(4):1406-14.
14. Koene R, van Liebergen F, Wijdeveld P. Normal diurnal rhythm in the excretion of water and electrolytes after renal transplantation. Clin Nephrol 1973;1(4):266-70.

15. Firsov D, Bonny O. Circadian regulation of renal function. Kidney Int 2010;78(7):640-5.

16. Cotes PM, Brozovic B. Diurnal variation of serum immunoreactive erythropoietin in a normal subject. Clin Endocrinol (Oxf) 1982;17:419-22.

17. Wide L, Bengtsson C, Birgegård G. Circadian rhythm of erythropoietin in human serum. Br. J. Haematol. 1989;72(1):85-90.

18. Klausen T, Poulsen TD, Fogh-Andersen N, Richalet JP, Nielsen OJ, Olsen N V. Diurnal variations of serum erythropoietin at sea level and altitude. Eur J Appl Physiol Occup Physiol 1996;72(4):297-302.

19. Miller ME, Garcia JF, Cohen R a, Cronkite EP, Moccia G, Acevedo J. Diurnal levels of immunoreactive erythropoietin in normal subjects and subjects with chronic lung disease. Br. J. Haematol. 1981;49(2):189-200.

20. Roberts D, Smith DJ. Erythropoietin does not demonstrate in healthy men circadian rhvthm. J Appl Physiol 1996;80(3):847-51.

21. Gunga H, Kirsch K, Baartz F, et al. Erythropoietin under real and simulated microgravity conditions in humans. J Appl Physiol 1985;81(2):761-73.

22. Böning D, Rojas J, Serrato M, et al. Hemoglobin mass and peak oxygen uptake in untrained and trained residents of moderate altitude. Int J Sports Med 2001;22(8):572-8.

23. Refinetti R, Lissen GC, Halberg F. Procedures for numerical analysis of circadian rhythms. Biol Rhythm Res [Internet] 2007 [cited 2013 Nov 11];38(4):275-325.

24. Revelle W. psych: Procedures for Personality and Psychological Research, NorthwesternUniversity. 2013;

25. Team RC. R: A language and environment for statistical computing. 2013;

26. Jammalamadaka SR, Sengupta A. Topics in Circular Statistics. $1^{\text {st }}$ ed. World Scientific Pub Co Inc; 2001. 336 p.

27. Agostinelli C, Lund U. R package "circular": Circular Statistics (version 0.4-7). 2013;

28. Hammer $\varnothing$, Harper D, Ryan P. Paleontological statistics software package for education and data analysis. Palaeontol Electron 2001;4(1):9.

29. Atkinson G, Coldwells A, Reilly T, Waterhouse J. A comparison of circadianrhythms in work performance between physically active and inactive subjects. Ergonomics. 1993 JanMar;36(1-3):273-81.

30. Ruff C. Variation in human body size and shape. Annu. Rev. Anthropol. 2002. 31:211-32.

31. Cheng CK-W, Chan J, Cembrowski GS, van Assendelft OW. Complete blood count reference interval diagrams derived from NHANES III: stratification by age, sex, and race. Lab Hematol [Internet] 2004 [cited 2013 Dec 1];10(1):42-53.

32. Saxena S, Wong ET. Heterogeneity of common hematologic parameters among racial, ethnic, and gender subgroups. Arch Pathol Lab Med [Internet] 1990 [cited 2013 Dec 1];114(7):715-9.

33. Nelson W, Tong Y, Lee J, Halberg F. Methods for cosinor-rhythmometry. Chronobiologia 1979;6(4):305-23. 
34. Pasqualetti P, Casale R. No influence of aging on the circadian rhythm of erythropoietin in healthy subjects. Gerontology 1997;43(4):206-9.

35. Cotes PM. The Estimation of Erythropoietin (Epo): Principles, Problems and Progress. In: Molecular and Cellular Aspects of Erythropoietin and Erythropoiesis. Springer Berlin Heidelberg; 1987. p. 377-87.

36. Fitzpatrick MF, Mackay T, Whyte KF, et al. Nocturnal desaturation and serum erythropoietin: a study in patients with chronic obstructive pulmonary disease and in normal subjects. Clin Sci (Lond) 1993;84(3):319-24.

37. Pasqualetti P, Collacciani a, Casale R. Circadian rhythm of serum erythropoietin in multiple myeloma. Am $\mathrm{J}$ Hematol 1996;53(1):40-2.
38. Stow LR, Gumz ML. The circadian clock in the kidney. J Am Soc Nephrol 2011;22(4):598-604.

39. Anderson GB, Bell ML, Peng RD. Methods to calculate the heat index as an exposure metric in environmental health research. Environ Health Perspect [Internet] 2013 [cited 2013 Dec 2];121(10):1111-9.

40. Halonen JI, Zanobetti A, Sparrow D, Vokonas PS, Schwartz J. Relationship between outdoor temperature and blood pressure. Occup Environ Med [Internet] 2011 [cited 2013 Dec 2];68(4):296-301.

41. Wallach T, Schellenberg K, Maier B, et al. Dynamic circadian protein-protein interaction networks predict temporal organization of cellular functions. PLoS Genet 2013;9(3):e1003398. 